Pontifícia Universidade Católica do Rio de Janeiro

Augusto da Cunha Reis

Gestão da variedade de produtos na indústria automobilística mundial

Dissertação de Mestrado

Dissertação apresentada como requisito parcial para obtenção do título de Mestre pelo Programa de PósGraduação em Engenharia de Produção da PUCRio.

Orientador: Prof. Luiz Felipe R. R. Scavarda do Carmo

Rio de Janeiro, março de 2009. 
Pontifícia Universidade Católica

do Rio de Janeiro

Augusto da Cunha Reis

\title{
Gestão da variedade de produtos na indústria automobilística mundial
}

\begin{abstract}
Dissertação apresentada como requisito parcial para obtenção do título de Mestre pelo Programa de PósGraduação em Engenharia de Produção da PUC-Rio. Aprovada pela Comissão Examinadora abaixo assinada.
\end{abstract}

Prof. Luiz Felipe R. R. Scavarda do Carmo Orientador Departamento de Engenharia Industrial - PUC-Rio

Prof. Osvaldo Luiz Gonçalves Quelhas Departamento de Engenharia de Produção - UFF

Prof. Madiagne Diallo Departamento de Engenharia Industrial - PUC-Rio

Prof. José Eugenio Leal Coordenador Setorial do Centro Técnico Científico - PUC-Rio

Rio de Janeiro, 09 de março de 2009. 
Todos os direitos reservados. É proibida a reprodução total ou parcial do trabalho sem autorização da universidade, do autor e do orientador.

\section{Augusto da Cunha Reis}

Graduou-se em Administração de Empresas pela PUC-Rio. Durante a graduação, estagiou na empresa Sistema FIRJAN (Federação das Indústrias do Estado do Rio de Janeiro) e na Frangos RICA (Reginaves Indústria e Comércio de Frangos Ltda) atuando no setor de suprimentos. Depois de graduado ingressou no Programa de Pós-graduação em Engenharia Industrial da PUC-Rio para obtenção do título de Mestre. Nesse período, trabalhou como pesquisador da PUC-Rio no Projeto ILIPT.

Ficha Catalográfica

Reis, Augusto da Cunha

Gestão da variedade de produtos na indústria automobilística mundial / Augusto da Cunha Reis ; orientador: Luiz Felipe R. R. Scavarda do Carmo. - 2009.

$97 \mathrm{f.} ; 30 \mathrm{~cm}$

Dissertação (Mestrado em Engenharia Industrial)Pontifícia Universidade Católica do Rio de Janeiro, Rio de Janeiro, 2009.

Inclui bibliografia

1. Engenharia Industrial - Teses. 2. Variedade de produtos. 3. Indústria automobilística. 4. Cadeia de suprimentos. 5. Postergação. 6. Compartilhamento de módulos. I. Carmo, Luiz Felipe Roris Rodriguez Scavarda do. II. Pontifícia Universidade Católica do Rio de Janeiro. Departamento de Engenharia Industrial. III. Título.

CDD: 658.5 
A meus pais, que sempre me deram apoio e segurança para realizar meus sonhos. 


\section{Agradecimentos}

A toda minha família, em particular aos meus amados pais, Marco Aurélio e Eloy, pelas alegrias compartilhadas, por todo carinho, compreensão, incentivo, confiança e ensinamentos, e a minha avó Alvina, pela força sempre transmitida e ainda tão presente em minha vida.

Ao meu Orientador e amigo Luiz Felipe Scavarda, pela atenção, confiança, segurança, motivação e conhecimentos transmitidos, desde a iniciação científica até hoje.

Aos funcionários da ANFAVEA, Aparecida e Cláudia, pela atenção e dedicação no fornecimento dos dados e pela excepcional recepção.

À Larissa Faria, pelo sorriso, carinho, companheirismo e amor, sempre presente ao meu lado, em todos os momentos da minha vida.

Aos profissionais do Projeto ILIPT, Leonardo Rainho e Tiago Fernandes, pela amizade e importantes contribuições para o desenvolvimento deste trabalho.

A Eduardo Salvino e Lucas Faria, que me ajudaram na recuperação dos dados.

Aos professores e funcionários do DEI da PUC-Rio, pelo apoio e infra-estrutura.

A todos os meus colegas de turma, especialmente Rafael Ferraz, Joana Gomes, Rafael Menezes, Mário Oliveira, Jin Wook e Débora Petroni, que acompanharam de perto todo caminho percorrido durante o Mestrado, ajudando a superar os desafios e comemorando comigo as vitórias conquistadas.

A meus grandes amigos (irmãos) Rodrigo Secchin e Pedro Fonseca, e a todos aqueles que de alguma forma contribuíram para a realização deste trabalho.

Ao CNPq, pela ajuda financeira concedida durante o curso. 


\section{Resumo}

Reis, Augusto da Cunha; Scavarda, Luiz Felipe R.R.. Gestão da variedade de produtos na indústria automobilística mundial. Rio de Janeiro, 2009. 97 p. Dissertação de Mestrado - Departamento de Engenharia Industrial, Pontifícia Universidade Católica do Rio de Janeiro.

A proliferação da variedade de produtos é uma tendência em diferentes indústrias e se constitui em um assunto com crescente relevância para o mundo empresarial e acadêmico. A gestão dessa variedade deve buscar equilibrar os benefícios oriundos da perspectiva do marketing com os efeitos negativos provocados, em geral, pela perspectiva de operações e logística. Assim, tal gestão se torna um grande desafio para indústrias em que a variedade de produtos é elevada, como a automobilística. O presente trabalho é um estudo empírico que tem como objetivo quantificar, comparar e analisar o comportamento da variedade de produtos nos mais significativos mercados automobilísticos mundiais. Como objeto de estudo, utilizou-se automóveis de passeio fabricados pelos cinco maiores grupos de montadoras (Toyota, GM, Volkswagen, Ford e Honda) e como variáveis da variedade de produtos na indústria automobilística, considerou-se plataformas, marcas, modelos, versões, tipos de carrocerias, motorização, transmissão (power-trains), pintura e acabamento interno (paint \& trim), opcionais de fábrica (factory fitted options) e acessórios de concessionárias (dealer fitted options). Como resultado, verificou-se em que ponto da cadeia automotiva ocorre a proliferação de produtos e analisou-se a forma distinta com que esta variedade é gerida em diferentes mercados a jusante da cadeia e a partir da fábrica de automóveis.

\section{Palavras-chave}

variedade de produtos, indústria automobilística, cadeia de suprimentos, postergação, compartilhamento de módulos 


\section{Abstract}

Reis, Augusto da Cunha; Scavarda, Luiz Felipe R.R.. Product variety management in automotive industry worldwide. Rio de Janeiro, 2009. 97 p. M. Sc. Dissertation - Departamento de Engenharia Industrial, Pontifícia Universidade Católica do Rio de Janeiro.

Product variety is a trend in many different industries and it is a subject with an increasing relevance to the business and academic arena. The management of product variety must seek to balance the benefits from the marketing perspective with the negative effects, in general, from the operations and logistics perspective, thus becoming a major challenge for industries where product variety is higher, such as the auto industry. This master's dissertation is an empirical study that aims to quantify, compare and analyze the behavior of the variety of products in the most significant global automobile markets. Passenger cars manufactured by the five largest groups of automakers in the world (Toyota, GM, Volkswagen, Ford and Honda) and variables of product variety in the auto industry such as platforms, brands, models, versions, body type, power-trains, paint \& trim, factory fitted options and dealer fitted options were used as objects of study. As a result, it was verified in which point of the supply chain the proliferation of automotive products occurs and the distinct way in which this variety is managed is analyzed in different markets downstream the chain from the car factory.

\section{Keywords}

product variety, auto industry, supply chain, postponement, component sharing 


\section{Sumário}

1 INTRODUÇÃO

2 REVISÃO BIBLIOGRÁFICA

2.1. VARIEDADE DE PRODUTOS: DEFINIÇÃO, IMPORTÂNCIA E VARIÁVEIS 13

2.2. DIMENSÕES DA VARIEDADE DE PRODUTOS

2.3. PROLIFERAÇÃO DE PRODUTOS 19

2.4. DIFERENTES PERSPECTIVAS SOBRE A VARIEDADE DE PRODUTOS 22

2.5 ESTRATÉGIAS DE MITIGAÇÃO

2.5.1 Modularização ou compartilhamento de componentes 24

2.5.2 Flexibilidade 30

2.5.3 Postergação $\quad 32$

2.6 HETEROGENEIDADE DA VARIEDADE DE PRODUTOS 33

3 MÉTODO DE PESQUISA

3.1 IMPORTÂNCIA DA INDÚSTRIA AUTOMOBILÍSTICA E SEUS SISTEMAS PRODUTIVOS 35

3.2 TIPOS DE VARIEDADE DE PRODUTOS NA INDÚSTRIA AUTOMOTIVA 38

3.3 INDÚSTRIA AUTOMOTIVA (SURVEY)

3.3.1 Coleta e processamento de dados (survey)

3.4 ESTUDO DE CASO (FORD) 43

3.4.1 Coleta e processamento de dados (caso Ford) 44

4 SURVEY NA INDÚSTRIA AUTOMOTIVA

$\begin{array}{ll}4.1 \text { AS EMPRESAS } & 48\end{array}$

4.1.1 Toyota 48

4.1.2 General Motors 49

4.1 .3 Volkswagen $\quad 50$

4.1.4 Ford 50

4.1.5 Honda 51

4.2 RESUlTADOS $\quad 51$

5 ESTUDO DE CASO - FORD $\quad 64$

5.1 OPCIONAIS DE FÁBRICA $\quad 64$

5.2 ACESSÓRIOS

5.3 VARIEDADE DE PRODUTOS PARA O FORD FIESTA HATCHBACK 76

6 CONCLUSÕES E CONSIDERAÇÕES FINAIS

7 REFERÊNCIAS BIBLIOGRÁFICAS

8 APÊNDICE $\quad 93$ 


\section{Lista de figuras}

FIGURA 1: VARIEDADE DINÂMICA PARA A AUDI EM 1980 E 2003.

FIGURA 2: VARIEDADE DINÂMICA PARA A ILUMINAÇÃO INTERNA DO AUDI C3 E C4. 17

FIGURA 3: VARIEDADE DE ILUMINAÇÃo INTERNA PARA AUdi A6. 18

FIGURA 4: ALTERAÇÃO NO OPCIONAL DE ILUMINAÇÃO INTERNA PARA O MODELO AUDI A6. 19

FIGURA 5: ESTRUTURA DA CADEIA DE SUPRIMENTOS DA INDÚSTRIA AUTOMOBILÍSTICA. 37

FIGURA 6: PRODUÇÃO DOS CINCO MAIORES GRUPOS DE MONTADORAS DO MUNDO. 41 


\section{Lista de tabelas}

TABELA 1: VARIEDADE DE PRODUTOS EM DIFERENTES INDÚSTRIAS 14

TABELA 2: ATRIBUTOS E VARIÁVEIS DA VARIEDADE DE PRODUTO 15

TABELA 3: VARIEDADE DE PRODUTOS SOB A PERSPECTIVA DE MARKETING 22

TABELA 4: VARIEDADE DE PRODUTOS SOB A PERSPECTIVA DE OPERAÇÕES E LOGÍSTICA 23

TABELA 5: FAMÍLIA DE MODELOS DO GRUPO VW COM A MESMA PLATAFORMA. 29

TABELA 6: MODELOS UTILIZADOS NO ESTUDO DE CASO DA FORD 43

TABELA 7: VARIEDADE DAS CINCO MAIORES NOS PRINCIPAIS MERCADOS

TABELA 8: VARIEDADE GRUPO FORD NOS PRINCIPAIS MERCADOS

TABELA 9: VARIEDADE GRUPO GM NOS PRINCIPAIS MERCADOS

TABELA 10: VARIEDADE GRUPO VOLKSWAGEN NOS PRINCIPAIS MERCADOS

TABELA 11: VARIEDADE GRUPO TOYOTA NOS PRINCIPAIS MERCADOS

TABELA 12: VARIEDADE GRUPO HONDA NOS PRINCIPAIS MERCADOS

TABELA 13: VARIEDADE DAS CINCO MAIORES PARA A UNIÃO EUROPÉIA

TABELA 14: VARIEDADE DAS CINCO MAIORES PARA OS PAÍSES EMERGENTES 58

TABELA 15: VARIEDADE DAS CINCO MAIORES PARA BRIC / BRICS / BRICM 59

TABELA 16: VARIEDADE DAS CINCO MAIORES PARA O G7 6

TABELA 17: VARIEDADE DAS CINCO MAIORES PARA OS SEGMENTOS DE MERCADO 63

TABELA 18: OPCIONAIS DE FÁBRICA - FORD KA

TABELA 19: OPCIONAIS DE FÁBRICA - FORD FIESTA HATCH 66

TABELA 20: OPCIONAIS DE FÁBRICA - FORD FIESTA SEDAN

TABELA 21: OPCIONAIS DE FÁBRICA - FORD FIESTA VAN $\quad 68$

TABELA 22: OPCIONAIS DE FÁBRICA - FORD FIESTA COURIER $\quad 68$

TABELA 23: OPCIONAIS DE FÁBRICA - FORD IKON / STREET

TABELA 24: OPCIONAIS DE FÁBRICA - FORD ECOSPORT / FUSION $\quad 70$

TABELA 25: OPCIONAIS DE FÁBRICA - FORD FOCUS HATCH 71

TABELA 26: OPCIONAIS DE FÁBRICA - FORD FOCUS SEDAN

TABELA 27: OPCIONAIS DE FÁBRICA - FORD FOCUS SW 72

TABELA 28: OPCIONAIS DE FÁBRICA - FORD FUSION

TABELA 29: OPCIONAIS E ACESSÓRIOS PARA O FIESTA HATCH $\quad 73$

TABELA 30: OPCIONAIS E ACESSÓRIOS PARA O FOCUS HATCH

TABELA 31: VARIEDADE PARA FIESTA EM MERCADOS SELECIONADOS

TABELA 32: DISPONIBILIDADE DE OPCIONAIS EM DIFERENTES MERCADOS 78 